\title{
SEMÁNTICA COGNITIVA DE LA METÁFORA QUECHUA
}

\section{UN ESTUDIO EN DISCURSOS DE LA VARIEDAD CUZCO-COLLAO}

\author{
RESUMEN \\ El siguiente trabajo de investigación, tiene el objetivo de \\ analizar, describir y explicar el fenómeno lingüístico y la \\ proyección de significados desde una perspectiva \\ semántico-cognitiva. El cognitivismo y el estudio de la \\ metáfora en el quechua comprende una propuesta que \\ pretende explicar el complejo panorama del \\ pensamiento cotidiano del hombre y su interacción con \\ el lenguaje, sosteniendo que el pensamiento tiene un \\ aspecto primordialmente metafórico e implica procesos \\ que no se desligan de la experiencia corpórea. En tal \\ sentido, se incide en el examen de los fenómenos \\ cognitivos desde la percepción de los hechos, el \\ movimiento corporal y la experiencia física y social del \\ hombre que se resume en las propiedades gestálticas \\ del lenguaje, observadas en discursos del quechua \\ Cuzco-Collao. Desde un enfoque cualitativo y \\ etnográfico de la investigación, se proponen \\ explicaciones conceptuales e inferenciales de las \\ fuentes escritas, orales y fonográficas seleccionadas \\ para este propósito. Cabe aclarar que los argumentos
}

Mario Ramos Tacca $^{1}$

expuestos en este trabajo, se distancian de las posturas estructuralistas saussureanas, es decir, no se basan en el estudio de la metáfora como artificio retórico o de recurso literario; por el contrario, sugiere un abordaje conceptual de alto nivel de abstracción para la comprensión de los complejos procesos del fenómeno lingüístico en su realización cotidiana.

PALABRAS CLAVE: Lengua, metáfora, semántica, cognitivismo, quechua.
ABSTRACT
This research main purpose is describe, analyze, and explain linguistic phenomenon to know the meanings since the cognitive and semantic perspective. Cognitive semantic and the study of metaphor in the Quechua language, is an approach that tries to explain the complex structure of the daily thinking of man. Cognitivism explain us that it has a primarily metaphorical aspect and involves processes that are not detached from the corporeal experience. Thereby, it redounds about the facts to explain cognitive

1 M.Sc. Lingüística Andina y Educación. Universidad Nacional de San Antonio Abad del Cusco. E-mail: siwarqinti1@gmail.com. 
phenomena since the perception of the facts, body movement, physical and social experience of man which is resume in the gestalt properties of language. Trough the qualitative research procedures and etnographic perspective, the analysis of the sentences contained in fragments of speeches from the Quechua of Cuzco-Collao varietyare approached to reach conceptual and inferential explanations arise in the projection of meaning located in each of the written, oral and phonograph selected statements for this purpose. Moreover, the reasons exposed in this work, take distance themselves from the saussureans and structuralist current positions. So, this work are not focussed on the study of metaphor as a rhetorical or literary resource, by the opposite, it suggests the develop of a conceptual work of high level abstraction to understand the complex processes of linguistic phenomenon in the daily performance.

Keywords: Language, metaphor, semantic, cognitivism, quechua.

"La moderna investigación

lingüística basada en el

cognitivismo, intenta

descubrir los contenidos

reales de la cognición

humana y los fenómenos

que habían sido ignorados

por muchos lingüistas."

$\mathrm{C}$

on el surgimiento de la Lingüística

Cognitiva como eje orientador de las ciencias cognitivas y tomando como fundamento los postulados de la misma, el presente estudio se rige a través de los aportes de la semántica cognitiva: proceso a través de los cuales se crea conciencia sobre el mundo en el que interactuamos.

El lenguaje figurado, las representaciones mentales, los conceptos, los esquemas y los modelos cognitivos idealizados son caracterizaciones del fenómeno conceptual en la realización lingüística. En consecuencia, el cognitivismo nos alcanza un aporte teórico-científico que plantea el estudio del lenguaje no puede separarse de su función cognitiva y menos comunicativa, este hecho evidencia que el enfoque basa sus fundamentos en los puntos de vista direccionados a describir las cualidades gestálticas de la lengua, en este caso: el quechua, que proporciona evidencias lingüístico-empíricas a través del cual, se concluye que nuestro pensamiento cotidiano es primordialmente metafórico y no se encuentra desligado de la experiencia corpórea (Nubiola, 1999).

De este modo, la moderna investigación lingüística basada en el cognitivismo, intenta descubrir los contenidos reales de la cognición humana y los fenómenos que habían sido ignorados por muchos lingüistas. De modo que esta tendencia basa sus postulados en el principio de que las categorías lingüísticas no son autónomas respecto de su organización conceptual general y a los mecanismos de procesamiento. Al contrario, se puede decir que esta nueva postura adopta un punto de vista filosófico que por algunos estudiosos como Lakoff y Johnson (1996) ha sido denominado como experiencialismo o realismo experiencial.

De acuerdo a los antecedentes teórico científicos, fue Max Black (1966) y Nelson Goodman (1968) quienes propusieron una visión distinta de metáfora. La postura de estos investigadores fundamenta sus explicaciones en la idea de que cuando usamos una metáfora hacemos uso de una sola expresión con dos formas de pensamiento de cosas distintas en actividad simultánea; cuyo significado final es el resultado de la interacción de los dos elementos.

Para Black los dos elementos vendrían a ser: uno el foco de la metáfora (el enunciado efectivo)y otro, el marco que lo rodea. En términos similares Lakoff 
(1980) identifica estos dos elementos con el nombre de dominios: dominio fuente y dominio destino. Es según esta teoría, que el segundo dominio se considera como un sistema más que como una cosa individual. Cuando decimos que la sociedad es un mar, estamos poniendo delante de nuestros ojos, proyectando sobre la sociedad, todo un sistema conceptual en el que hay tempestades, puertos seguros, piratas, tiburones, naufragios y muchas cosas más (Cuenca \& Hilferty, 1999).

Del mismo modo, cuando en quechua decimos: kay simiypuni huchayuq (mi boca tiene la culpa), kay ñawiy huchayuq (mis ojos tienen la culpa) estamos desplegando toda una gama de connotaciones conceptuales que asocian nuestro modo de concebir el mundo en términos ético-morales con las experiencias corpóreas del fenómeno lingüístico.

En consecuencia, la lengua quechua y su particular forma de realización cotidiana es la que ha despertado mucha curiosidad entre los estudiosos y lingüistas contemporáneos, pues la aplicación de este nuevo enfoque, permite describir el fenómeno figurativo desde la perspectiva de la relación entre pensamiento y lenguaje observado en fragmentos metafóricos obtenidos a partir de textos dialógicos, fonográficos y escritos diversos de origen quechua.

Por lo mismo, a través de los aportes de la semántica cognitiva también se puede aseverar que la metáfora o acto connotativo del lenguaje no es prioridad de la literatura, sino que este fenómeno se observa en el uso del lenguaje cotidiano y todos los niveles de cualquier lengua. Consiguientemente, se concluye que todos los ámbitos comunicativos del quechua también se encuentran plagados de expresiones cognitivo-connotativas que lo tornan más agradable y vivazen su realización cotidiana.
De ello podemos derivar que el enfoque interactivo de la metáfora supone un cambio importante de atención: en lugar de atender a las metáforas como realizaciones o productos de la actividad artística, han pasado a ser estudiadas, ahora, como procesos de construcción de significados. Este cambio (que en lingüística significa un giro de nuestra atención desde la semántica a la pragmática) se debe en buena cuenta a la moderna revolución cognitiva que traspasa los límites tradicionales de las disciplinas en busca de una cabal comprensión de la inteligencia humana. Por ello, para nuestro interés, se ha propuesto un conjunto de preguntas que esta investigación pretende resolver: ¿De qué manera se determina el carácter cognitivo-conceptual de la metáfora en los discursos quechua de la variedad Cuzco-Collao?, ¿Cómo se describen los niveles significativos del quechua en la proyección metafórica cognitiva?, ¿De qué manera se analiza la estructura cognitiva de la metáfora quechua en base a sus rasgos semánticos?, ¿Cómo se describen los componentes significativo-experienciales del quechua en la proyección metafórica?

No obstante, dentro de los distintos campos significativos que caracterizan a las lenguas, en cuanto al quechua, lo fundamental para el presente estudio es relevar la incidencia de la metáfora en la construcción de significados contextuales, pues muchas de ellas son generadas por estructuras básicas de nuestra experiencia y nuestra manera particular de pensar. Buena parte de la coherencia y el orden de nuestra actividad conceptualizadora se basa en el modo en que nuestros sistemas de metáforas estructuran la experiencia.

Para ejemplificar el tema, a continuación obsérvese el siguiente fragmento metafórico quechua extraído de Büttner \& Chuquimamani (1986) con sus respectivas descripciones e interpretaciones cognitivo-conceptuales: 


\section{EL CLIMA ES UNA ENTIDAD HUMANA}

$<$ Kay ayllunchiskunapi,
mana ch'akiriqtinqa qasañataq
mikhuyninchista aparikapun>

(En nuestras comunidades, sino es la sequía, es la helada la que se lleva nuestros alimentos)

Al efectuar un análisis descriptivo de los elementos cognitivos del fragmento y sus correlatos ontológicos, se observa que éste posee un alto contenido metafórico dentro de su estructura semántica. A primera vista, se puede observar la concepción peculiar del mundo que tiene el hablante, seguido de la relación existente entre el hombre y la naturaleza que lo circunda. Sin embargo, no es menos atinado, también, observar que la base de su significado cognitivo tiene asidero en la experiencia de la vida cotidiana que invita a conocer ese entramado de conceptos, comprensión e interpretación de la compleja realidad sociocultural y lingüística del hablante quechua. En consecuencia, hombre y naturaleza aparecen como el binomio complementario perfecto que interactúa en un espacio cósmico a través de las distintas etapas de los ciclos agrícolas y productivos como la siembra, la cosecha, aporque, riego, cultivo de plantas, gastronomía e idiosincrasia propias del mundo andino. Por ello, a través de este vínculo, el hombre despliega toda una gama de conceptos cognitivos con base en la experiencia sensitiva en el dominio concreto y abstracto. Es por ello, que a través del dominio de los sentimientos y el trasfondo experiencial, los factores climatológicos adquieren vida, pueden adoptar conductas benévolas y/o perversas como castigar y arrebatar los alimentos provocando padecimientos en la gente.
Con todo este antecedente, se puede advertir que el trabajo de investigación contribuye a la explicación de los complejos fenómenos de construcción de conceptos cognitivos en el quechua, partiendo de presupuestos metafóricos $\mathrm{y}$ filosóficos con base en los planteamientos de la psicología de la gestalt.

Finalmente, creemos conveniente anotar que enfocar un tema de investigación de esta naturaleza, en sus bases, procura ser un aporte a los estudios de la Lingüística Andina, específicamente en el campo de la interpretación del discurso cognitivo y de los estudios lingüísticos del quechua Cuzco-Collao.

\section{MÉTODOS}

Considerando que el propósito fundamental de toda investigación es alcanzar soluciones a los problemas desarrollando procesos epistemológicos, nos valimos del trabajo de campo y la observación que en un primer momento, nos permitió recopilar el material de trabajo, en el que se describe, analiza y discute expresiones metafóricas, habladas y escritas, provenientes del quechua de la variedad Cuzco-Collao. De modo que el proceso comprende los siguientes pasos:

-a) La metodología corresponde a la investigación cualitativa dentro del campo de las ciencias sociales, hecho que nos ofrece un flexible y amplio panorama de posibilidades que permite buscar soluciones y respuestas al problema a través de procedimientos propios sobre los fenómenos sociales desde la perspectiva del actor.

-b) El tipo de investigación sigue una secuencia descriptiva que corresponde a la etnografía: estudio a través del cual se aprende el modo de vida de la unidad social. También a través de la etnografía se persigue la descripción y la reconstrucción analítica del carácter 
interpretativo de la cultura, sus formas de vida y la estructura social del grupo investigado (Sampieri, 2009).

-c) El tipo de muestra es criterial, focalizada en los ámbitos geográficos de las regiones de Cuzco y Puno, específicamente las zonas limítrofes de habla quechua comprendidas en las provincias altas del Cuzco y la zona norte de Puno, de las cuales, se seleccionó diez (10) canciones vernaculares, tres (03) textos de tipología variada escritos en quechua más dos (02) programas radiales en quechua del ámbito de Puno, haciendo un total de quince (15) fuentes orales y escritas.

-d) La técnica corresponde al análisis de contenido, que permitió describir los significados de los documentos o mensajes sean estos: un discurso, una historia de vida, un artículo de revista, un texto escolar, un decreto ley, en fin, un corpus que tenga la cualidad de ser textual, transcrita o un material de tipo fonográfico-audiovisual.

-e) Los instrumentos de recolección de datos comprende: el uso del cuaderno de campo, la ficha de registro de canciones por áreas de difusión, las fichas de transcripción fonográfica, escrita y radial de los textos quechua (Montoya, 1987).

-f) El procedimiento y análisis que se siguió en la sistematización de los datos comprende, en primer lugar: el análisis y la metaforización de fragmentos quechua extraídos de discursos escritos, diálogos y canciones vernaculares contemporáneas caracterizadas a través de dominios de experiencias sensoriales, motoras y cognitivas con base en las pautas y modelos conceptuales esbozados especialmente por Lakoff, Jonson y Turner y una adaptación del modelo para la descripción y análisis de la metáfora propuesta por Rivano (1999).

\section{RESULTADOS Y DISCUSIONES}

Como anotamos anteriormente, la preocupación fundamental del presente estudio es demostrar que la metáfora enfocada desde una perspectiva semántico-cognitiva coadyuva a entender cómo creamos sentido a nuestro alrededor y cómo ésta nos permite subsistir en el mundo en el que vivimos e interactuamos creando ámbitos de referencia entre mundo y sujeto, considerando que éstos no son procesos de creación de sentido separables y distinguibles, sino que ambos confluyen para la edificación de nuevas metáforas que describen el proceso por el cual las palabras remiten a las cosas.

En consecuencia, para alcanzar una respuesta a los ítems planteados y el análisis semántico cognitivo del corpus seleccionado; Lakoff y Johnson (1996) sugieren la utilización de una propuesta descriptiva y de interpretación cognitiva cuyo marco teórico sostiene que la estructura interna de las metáforas conceptuales se analiza además siguiendo dos dominios: de origen y destino; pues según ello, la metáfora se entiende como la proyección de unos conceptos desde un dominio conceptual (dominio fuente) hacia otro dominio conceptual (dominio meta).

Para entender con mayor exactitud la estructura interna de las metáforas cognitivas, veamos los ejemplos de dominios cognitivos quechua y sus correspondencias propuestas a partir de las metáforas conceptuales: estructurales, orientacionales y ontológicas.

A) METÁFORAS ONTOLÓGICAS: Son aquellas por las que se categoriza un fenómeno de forma peculiar mediante su consideración como entidad, una sustancia, un recipiente, una persona, etc. 
1. Los SENTIMIENTOS son OBJETOS.

a) Dominio origen: LOS

SENTIMIENTOS; Dominio destino: LOS OBJETOS

$<$ Chay, chay

Yana ñawichaykis,

Sunquyta suwallawashan

Chay, chay

Puka sintachaykis

Sunquyta suwallawashan>

(Poesía Quechua Diglósica, 1994:59)

Esos tus ojitos negros son los que me roban el corazón. Esa cinta roja es la que me roba el corazón.

\section{Los OBJETOS son ENTIDADES}

\section{HUMANAS}

b) Dominio origen: ENTIDADES HUMANAS; Dominio destino: LOS OBJETOS

$<$ Chiwanway waytita q'umir rap'ischallay, ama ch'akinkichu

kutimunaykama, ama sisankichu wiltamunaykama. Lima llaqtamanta kutiramuspaña, Arikipa llaqtamanta wiltaramuspaña, phaña warasuchaypi apakapuchkayki, warma wiqichaywan ch'alla, ch'allaykuspa>

("Chiwanway Waytita" Qori Taki, 2008. Formato CD)

Verde flor de Chiwanwa no te marchites ni florezcas hasta mi retorno. Volviendo de Lima o Arequipa, te llevaré en mi brazo derecho, regándote con mis tiernas lágrimas.

La interpretación ontológica de la muestra (a) corresponde a la descripción: Los sentimientos son objetos. Por tanto, se proyecta una metáfora, donde se observa que ciertos órganos del cuerpo desempeñan funciones cleptomaniacas frente a los sentimientos amorosos. En (b) la flor silvestre corresponde a un ser animado con sentimientos.
B) METÁFORAS ORIENTACIONALES:

Organizan un sistema global de conceptos con relación a otro sistema. La mayoría de ellos tiene que ver con la orientación espacial y nacen de nuestra constitución física.

1. LA SOLTERÍA es ARRIBA; EL MATRIMONIO ES ABAJO.

(a) Dominio origen: LA SOLTERÍA; Dominio destino: EL MATRIMONIO

$<$ Imas ñuqari

kasarayman

alturaqsi inti

ñuqapaqqa $>$

(Poesía Quechua Diglósica, 1994:105)

Que voy a casarme, para mí es temprano aun.

2. Las RELACIONES PERSONALES son CONDUCTAS BUENAS (ARRIBA) Y MALAS (ABAJO)

(b) Dominio origen: RELACIONES

PERSONALES; Dominio destino: CONDUCTAS

$<$ Kunansi isu sí, t'aqanaykapusun.

Qamtaq urayman, ñuataq wichayman; mayqinchanllanchikchá yawarta waqasun>

("Seda pañuelo" Kempor de Cusipata, 1975. Formato CD)

Ahora, sí, nos separaremos. Tú, hacia abajo y, yo, hacia arriba. Ya veremos cuál de los dos llora sangre.

En la muestra siguiente, observamos que las correspondencias ontológicas en (a) proyectan un tipo de metáfora orientacional, donde la virtud subliminal de la soltería es ubicada en una esfera vertical de superioridad frente a la oposición inferencial de inferioridad del matrimonio como efecto restrictivo del goce de la libertad. En (b) ir en sentidos opuestos, uno para arriba y el otro para abajo corresponde a proyectar una 
inferencia de sufrimiento y llanto para quien baja.

C) METÁFORAS ESTRUCTURALES: En las que una actividad o una experiencia se estructura en términos de otra.

1. El TIEMPO es un SER con SENTIMIENTOS.

(a) Dominio origen: LA COLORACIÓN DE LA NUBE; Dominio destino: LOS SENTIMIENTOS

$<$ Qhawarinkichu
haqay phuyuta
k'ancharimuntaq
tutayarintaq
chay hinallataq
warmalla munasqay
yuyariwantaq
qunqariwantaq $>$

(Poesía Quechua Diglósica, 1994:108)

¿Ves aquella nube? Se ilumina y oscurece, asi el muchacho que amo, me recuerda y me olvida.

\section{Los PENSAMIENTOS son OBJETOS} FÍSICOS

(b) Dominio origen: LOS

PENSAMIENTOS; Dominio destino: LOS OBJETOS FÍSICOS

$<$ Chimpa urqumantas tuku waqamun, chimpa urqumantas ch'usiq waqamun. Ch'usiq waqamun, ch'usaq tukunaypaq. Tuku waqamun, tukuyukunaypaq>

("Quencha” Bohemios del Cuzco, 1980. Formato CD)

Del cerro de enfrente canta el búho, canta la lechuza. Canta la lechuza para desaparecer, canta el búho para acabar mi existencia.

La muestra (a), expresa la proyección semántica de una metáfora estructural, donde el fenómeno ontológico remite al recuerdo y el olvido del ser amado que son opacados por la coloración de la nube. En (b) las aves adquieren conductas racionales que juegan con el azar y corresponde a predecir la desgracia y la desventura de las personas.

\section{CONCLUSIONES}

En la investigación Semántica cognitiva de la metáfora quechua: un estudio en discursos de la variedad

$$
\begin{aligned}
& \text { "Estos componentes } \\
& \text { experienciales se evidencian } \\
& \text { en los procesos sensoriales } \\
& \text { y corpóreos cotidianos que } \\
& \text { juegan un rol } \\
& \text { preponderante en la } \\
& \text { construcción de sentido a } \\
& \text { nuestro alrededor." }
\end{aligned}
$$

Cuzco-Collao, luego de haber realizado el proceso de análisis, descripción, discusión e interpretación de los resultados se arribó a las siguientes conclusiones concordantes con los objetivos de investigación:

Primera. A través del estudio de los fragmentos del quechua Cuzco-Collao, se verifica y se comprueba que el carácter cognitivo del lenguaje permite conocer los complejos procesos conceptuales del hablante desde una perspectiva sensorial que se evidencia a través del estudio de la metáfora como mecanismo que involucra procesos del pensamiento, el lenguaje y la experiencia corpórea. 
Segunda. La metáfora, observada desde la perspectiva de la lingüística cognitiva, demuestra que los niveles significativos del quechua se pueden describir desde un enfoque semántico-cognitivo (dominios cognitivos) hecho que coadyuva a entender cómo, a través del lenguaje, la experiencia corpórea y el pensamiento, creamos sentido a nuestro alrededor y cómo ésta nos permite subsistir en el mundo en el que vivimos e interactuamos creando ámbitos de referencia entre mundo y sujeto, considerando que éstos no son procesos de creación de sentido separables y distinguibles, sino que ambos confluyen para la edificación de nuevas metáforas que describen el proceso por el cual las palabras remiten a las cosas.

Tercera. A través de los fragmentos analizados podemos entender que en el quechua el fenómeno metafórico es de realización cotidiana, lo que hace ver que los procedimientos cognitivos del lenguaje permiten desarrollar complejos procesos de análisis de los campos significativos del quechua y sus correspondencias ontológicas en discursos escritos y orales. La estructura cognitiva de la metáfora implica la elaboración de conceptos a través de nociones ontológicas, orientacionales y estructurales. Operaciones mentales que permiten explicar el tema desde una perspectiva gestáltica, que se expresa en la infinidad de contenidos metafóricos estudiados en los enunciados conceptuales analizados.
Cuarta. La semántica cognitiva a través de su marco teórico expuesto en los acápites precedentes, permiten describir científicamente los aspectos significativo-experienciales en la proyección de conceptos metafóricos desde una postura filosófica del lenguaje. Así, estos componentes experienciales se evidencian en los procesos sensoriales y corpóreos cotidianos que juegan un rol preponderante en la construcción de sentido a nuestro alrededor.

\section{REFERENCIAS}

Black, M. (1966). Models and Metaphors. Studies in Language and Philosophy. Cornell University Press, Ithaca. Consulta: 21 de abril de 2012. Disponible en: https://www.ucm.es/data/cont/docs/481-2013-10-14

Bütner, M. M., \& Chuquimamani, N. R. (1986). Yanamayu Ayllu 2. Cusco: Editorial Centro Bartolomé de las Casas.

Cuenca, M. J., \& Hilferty, J. (1999). Introducción a la lingüistica cognitiva. Barcelona: Ariel lingüística.

Cusihuaman, A. (1976). Gramática Quechua: Cuzco-Collao. Lima: Ministerio de Educación - Instituto de Estudios Peruanos - IEP.

Goodman. N. (1968) Los lenguajes del arte: aproximación a la teoría de los símbolos. Consulta: 17 de mayo de 2014. Disponible en: http://www.descargarlibro.org/ los-lenguajes-del-arte-nelson-goodman-libro.html

Hurtado de Mendoza, W. (1993). Poesía quechua diglósica. Lima: Editorial Universidad Nacional Agraria La Molina.

Lakoff, G., \& Jonson, M. (2009). Metáforas de la vida cotidiana. Madrid: Cátedra. ISBN: 9788437606330.

Montoya, R. (1987). La sangre de los cerros. Lima: Editorial Universidad Nacional Mayor de San Marcos.

Nubiola, J. (1999). El valor cognitivo de las metáforas. Cuadernos de Anuario Filosófico, 18(103), 73-84. Consulta: 11 de agosto de 2012. Disponible en: www.unav.es/user/ValorCognitivoMetafora.html

Rivano. E. (1999). Un modelo para la descripción y análisis de la metáfora. Revista LOGOS, 14(9), 41-51. Consulta: 12 de febrero de 2014. Disponible en: http://revistas. userena.cl/index.php/logos/article/viewFile/341/371

Sampieri, R. H., Fernández, C. \& Baptista, P. (2006). Metodología de la investigación. México: Mc Graw Hill 